\title{
Méthodes numériques de propagation de fissures appliquées au découpage des métaux
}

\author{
Vincent Lemiale*,** — Jérôme Chambert* \\ Abdelhamid Touache* — Philippe Picart* \\ * Université de Franche-Comté, Institut FEMTO-ST - UMR 6174 \\ Laboratoire de Mécanique Appliquée R. Chaléat \\ 24 rue de l'Epitaphe, F-25000 Besançon cedex \\ \{jerome.chambert, abdelhamid.touache,philippe.picart\}@univ-fcomte.fr \\ ** Monash University, School of mathematical sciences \\ Clayton campus building 28 \\ Clayton VIC 3800, Australia \\ Vincent.Lemiale@sci.monash.edu.au
}

\begin{abstract}
RÉSUMÉ. L'objectif du travail présenté est de proposer un outil numérique efficace pour simuler le procédé de découpage et pour déterminer les caractéristiques géométriques et mécaniques d'une pièce découpée. Deux stratégies distinctes sont proposées pour simuler la propagation de fissures : une méthode d'élimination des éléments finis et une approche de fissuration discrète. En premier lieu, ces deux méthodes ont été évaluées sur l'exemple-test d'une plaque asymétrique en traction. En second lieu, la capacité de ces deux méthodes de propagation à prédire un profil de rupture réaliste est analysée dans le cadre du découpage. Les courbes effort-déplacement du poinçon obtenues par ces deux méthodes de propagation de fissures sont comparées à des courbes expérimentales.

ABSTRACT. The purpose of this paper is to propose an efficient numerical tool to simulate the blanking process and predict the geometric and mechanical characteristics of the blanked component. Two different strategies are proposed to simulate the crack propagation: a finite element elimination method and a discrete cracking approach. First, these methods are evaluated on the set-up test of an asymmetrical plate submitted to traction. Second, the ability of these methods to predict a realistic cut edge profile is analyzed within the framework of blanking. The load - penetration punch curves obtained by both fracture propagation methods are compared to the experimental one.

MOTS-CLÉS : méthode des éléments finis, grandes déformations, remaillage, critère de rupture, propagation de fissure, méthode d'élimination des éléments, approche de fissuration discrète, découpage des métaux.

KEYWORDS: finite element method, large deformations, remeshing, fracture criteria, crack propagation, element elimination method, discrete fracture approach, metal blanking.
\end{abstract}

DOI:10.3166/REMN.16.889-911 @ 2007 Lavoisier, Paris

REMN - 16/2007. Modélisations numériques en mécanique, pages 889 à 911 


\section{Introduction}

Le procédé de découpage est très utilisé par les industries électronique et micromécanique pour produire en grande quantité des pièces de très petites dimensions. Actuellement, la conception et la fabrication de ces composants reposent encore pour une bonne part sur le savoir-faire des opérateurs. Or les besoins industriels actuels imposent de produire plus rapidement des pièces de plus en plus petites, constituées de matériaux variés, et possédant des propriétés mécaniques spécifiques avec un contrôle géométrique très précis. Dans la plupart des cas, l'approche empirique n'est plus suffisante.

Afin de prévenir les méthodes empiriques et de réduire les coûts de production, de nombreuses approches analytiques et numériques par éléments finis ont été développées par des scientifiques et des chercheurs pour modéliser le processus complet de découpage de tôle métallique. La première phase de ce processus correspond à un comportement élastique de la tôle. Dans la seconde phase, le comportement est élastoplastique avec ou sans écrouissage suivant le type de matériau. A partir d'un certain niveau de pénétration du poinçon, la déformation plastique se localise dans une bande de cisaillement située entre les rayons du poinçon et de la matrice. Parallèlement à l'augmentation de la déformation plastique, l'endommagement se développe progressivement dans cette bande, ce qui conduit à l'amorçage d'une ou plusieurs fissures macroscopiques au niveau des arêtes du poinçon et de la matrice. Dans la dernière phase, la (ou les) fissure(s) se propage(nt) pour conduire à la rupture complète de la tôle.

C'est l'ensemble de ces processus de déformation qu'il importe de modéliser pour traduire le plus fidèlement possible le comportement du matériau lors du découpage. Dans cet article, l'accent est mis plus particulièrement sur la simulation de la propagation de fissures. Dans le cadre du découpage des métaux par la méthode des éléments finis, cinq types d'approche ont été proposés dans la littérature pour simuler numériquement la propagation de fissures.

Certains auteurs modélisent l'évolution d'une fissure par la méthode d'annulation de la raideur (Maillard, 1991 ; Hambli, 1996). Lorsqu'un élément fini satisfait à un certain critère de rupture, l'élément est considéré comme rompu et sa contribution à la matrice de raideur globale est annulée. En pratique, une valeur infinitésimale mais non nulle lui est assignée pour éviter toute instabilité numérique. Ainsi, cet élément ne contribue plus au calcul global.

La seconde technique est la méthode d'élimination des éléments (Taupin et al., 1996 ; Ko et al.,1997 ; Fang et al., 2002). Lorsqu'un élément satisfait au critère de rupture préalablement défini, il est déclaré rompu et puis supprimé du maillage. Cette méthode est implémentée dans de nombreux codes commerciaux de calcul par éléments finis. La différence principale entre ces deux premières méthodes provient de la modification topologique du maillage pour la technique d'élimination. 
La technique de suppression des éléments entraîne une diminution de la masse globale de la structure et donc une certaine imprécision (Komori, 2001). Afin d'éviter ce problème, une troisième approche a été proposée: la méthode de séparation des nœuds. Lorsqu'un nœud satisfait au critère de rupture, il est dédoublé. Le relâchement local des contraintes en ce nœud permet d'ouvrir une fissure à cet endroit (Hatanaka et al., 2003 ; Komori, 2005).

Ces trois dernières méthodes comportent un inconvénient majeur, à savoir la dépendance au maillage par éléments finis. Brokken propose alors la méthode de fissuration discrète (Brokken, 1999 ; Brokken et al., 2000). Cette approche repose sur le fait qu'une fissure n'est pas astreinte à se propager suivant une arête de l'élément, comme dans la simple séparation des nœuds. Le chemin de propagation est déterminé suivant la direction définie par la valeur maximale du critère de rupture. Une étape de remaillage suit pour accommoder l'accroissement de la ligne de rupture.

Enfin, la dernière méthode est fondée sur la mécanique de la rupture. Il s'agit réellement de l'étude d'un milieu fissuré, par opposition avec les précédentes méthodes. Bouchard (2000) présente une application au cas du découpage des métaux en utilisant la mécanique non linéaire de la rupture (Bouchard et al., 2002). Cependant, le calcul n'est pas mené à son terme dans la mesure où des problèmes d'interpénétration des lèvres d'une fissure surviennent. Il s'agit d'une des rares applications de cette théorie au découpage.

Dans cet article, la modélisation du découpage est envisagée par une approche découplée, c'est-à-dire que l'endommagement n'influe pas sur le comportement mécanique de la tôle. Nous avons focalisé notre analyse sur la méthode d'élimination des éléments et sur l'approche de fissuration discrète. Ces deux méthodes de propagation de fissures ont été implémentées dans le code de calcul par éléments finis BLANKFORM développé au sein du laboratoire (Lemiale, 2004). Ces deux approches sont appliquées pour simuler par éléments finis, d'une part, un essai de traction d'une plaque asymétrique en déformations planes, et d'autre part, un essai de découpage axisymétrique. Les simulations numériques du découpage sont comparées à des résultats expérimentaux.

\section{Modélisation de la fissuration}

\subsection{Critères de rupture}

L'utilisation d'un critère de rupture ductile est l'une des méthodes permettant de détecter le lieu d'amorçage d'une fissure. Dans cet article, une approche non couplée est utilisée : une fonction $D$ représentative de l'état d'endommagement est introduite dans la modélisation sans que la dégradation progressive des propriétés mécaniques du matériau ne soit prise en compte. Cette fonction indicatrice de l'endommagement $D$ dépend de l'histoire des tenseurs des déformations $\varepsilon$ et des contraintes $\sigma$. Les critères de rupture s'écrivent alors sous la forme générale suivante : 


$$
D=\int_{0}^{\bar{\varepsilon}^{\mathrm{p}}(t)} f(\sigma) \mathrm{d} \bar{\varepsilon}^{\mathrm{p}} \leq D_{\mathrm{C}}
$$

où $\bar{\varepsilon}^{\mathrm{p}}$ est la déformation plastique équivalente. Dès que la fonction $D$ atteint une valeur critique $D_{\mathrm{C}}$, une fissure est supposée s'amorcer. $D_{\mathrm{C}}$ est un paramètre matériau qui peut être identifié par des méthodes directes ou inverses à partir de données expérimentales. La fonction $f$ de l'équation [1] représente une fonction scalaire du tenseur $\sigma$. Le tableau 1 présente une liste des critères de rupture les plus utilisés dans le domaine de la mise en forme. Ces critères ont été déterminés, soit de manière empirique, soit par des approches micromécaniques fondées sur la croissance d'une microcavité.

\begin{tabular}{|ll|}
\hline Référence/Type de modèle & Critère de rupture \\
\hline $\begin{array}{l}\text { (Freudenthal, 1950) } \\
\text { Modèle empirique }\end{array}$ & $D=\int_{0}^{\bar{\varepsilon}^{\mathrm{p}}} \bar{\sigma} \mathrm{d} \bar{\varepsilon}^{\mathrm{p}}$ \\
\hline $\begin{array}{l}\text { (Cockroft and Latham, 1968) } \\
\text { Modèle empirique }\end{array}$ & $D=\int_{0}^{\bar{\varepsilon}^{\mathrm{p}}} \sigma_{1} \mathrm{~d} \bar{\varepsilon}^{\mathrm{p}}$ \\
\hline $\begin{array}{l}\text { (McClintock, 1968) } \\
\text { Modèle micromécanique }\end{array}$ & $D=\int_{0}^{\bar{\varepsilon}^{\mathrm{p}}} \frac{2}{\sqrt{3}(1-n)} \sinh \left[\frac{\sqrt{3}}{2}(1-n) \frac{\sigma_{1}+\sigma_{2}}{\bar{\sigma}}\right] \mathrm{d} \bar{\varepsilon}^{\mathrm{p}}$ \\
\hline $\begin{array}{l}\text { (Rice and Tracey, 1969) } \\
\text { Modèle micromécanique }\end{array}$ & $D=\int_{0}^{\bar{\varepsilon}^{\mathrm{p}}} A \exp \left(\frac{3}{2} \frac{\sigma_{\mathrm{m}}}{\bar{\sigma}}\right) \mathrm{d} \bar{\varepsilon}^{\mathrm{p}}$ \\
\hline $\begin{array}{l}\text { (Oyane, 1980) } \\
\text { Modèle micromécanique }\end{array}$ & $D=\int_{0}^{\bar{\varepsilon}^{\mathrm{p}}}\left(1+A \frac{\sigma_{\mathrm{m}}}{\bar{\sigma}}\right) \mathrm{d} \bar{\varepsilon}^{\mathrm{p}}$ \\
\hline
\end{tabular}

Tableau 1. Critères de rupture ductile les plus utilisés en mise en forme

Dans le tableau 1, $\bar{\sigma}$ est la contrainte équivalente de Von Mises, $\sigma_{\mathrm{m}}$ la contrainte normale moyenne, $\sigma_{1} \geq \sigma_{2} \geq \sigma_{3}$ les contraintes principales, $A$ une constante matérielle à identifier et $n$ le coefficient d'écrouissage.

Une synthèse complète sur les critères de rupture dans le découpage de tôle peut être trouvée dans les références suivantes (Hambli, 1996; Wisselink, 2000; Lemiale, 2004). Ces études ont montré que les paramètres des critères de rupture ( $A$ et $D_{\mathrm{C}}$ ), qui devraient être des constantes matérielles, peuvent aussi dépendre du procédé, et donc du type de conditions aux limites. Dans le cadre d'une utilisation industrielle de BLANKFORM, la principale difficulté dans ce genre d'approche est donc la caractérisation de ces paramètres pour différents matériaux. 


\subsection{Méthode d'élimination des éléments}

La méthode d'élimination des éléments consiste à enlever du maillage les éléments finis qui satisfont à une valeur critique de l'endommagement. Les éléments endommagés sont simplement supprimés et ils ne contribuent plus au calcul de la matrice globale de rigidité. La figure 1 montre les différentes configurations pour lesquelles des éléments endommagés peuvent être ou non éliminés.
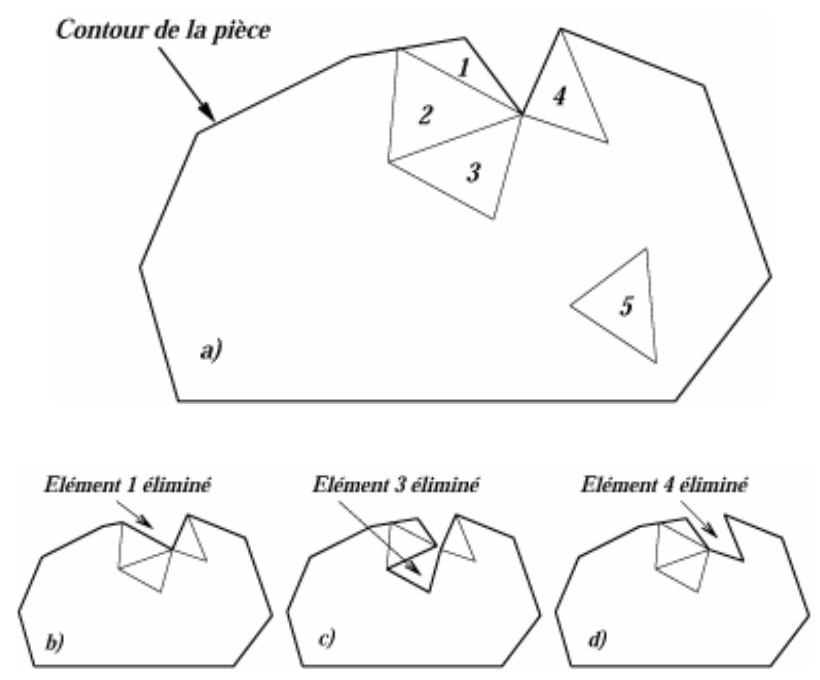

Figure 1. Méthode d'élimination des éléments

Cette méthode a été implémentée dans le code BLANKFORM (Lemiale, 2004) pour des éléments triangulaires sous les conditions suivantes :

- seuls les éléments dont au moins un nœud appartient au contour de la pièce peuvent être éliminés. Par conséquent, les fissures ne peuvent s'amorcer que sur les bords de la tôle. Il est donc impossible d'enlever l'élément 5 même si le critère est atteint (cf. figure 1a),

- si seulement un nœud de l'élément appartient au contour, alors ce nœud est dédoublé (en ajoutant un nœud supplémentaire sur le contour) et l'élément est enlevé du maillage (voir l'élément 3 sur la figure 1c),

- considérons le cas pour lequel deux ou trois nœuds de l'élément appartiennent au contour. Si ces nœuds sont consécutifs sur le contour, alors l'élément est supprimé (voir les éléments 1 et 4 sur les figures $1 \mathrm{~b}$ et $1 \mathrm{~d}$, respectivement), sinon l'élément n'est pas retiré (voir l'élément 2 sur la figure 1a). 


\subsection{Approche de fissuration discrète}

Cette méthode numérique, relativement proche de celle proposée dans (Brokken, 1999), a été incorporée dans BLANKFORM en considérant les trois étapes suivantes :

1. amorçage d'une fissure en utilisant un critère de rupture,

2. propagation ou non de la fissure initiée,

3. détermination du trajet de fissuration si propagation.

L’implémentation numérique doit respecter les restrictions suivantes :

- l'initiation d'une fissure n'est possible que sur le contour originel de la pièce,

- l'amorçage d'une nouvelle fissure n'est pas autorisé sur les lèvres d'une fissure existante,

- le calcul est arrêté si la pièce est divisée en deux parties ou si la distance entre les pointes des deux fissures atteint une valeur seuil.

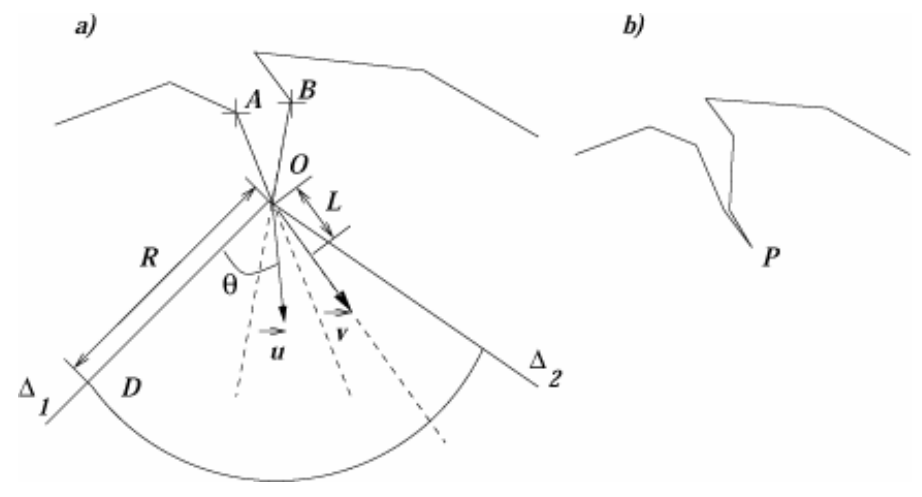

Figure 2. Approche de fissuration discrète

Les figures $2 \mathrm{a}$ et $2 \mathrm{~b}$ illustrent la propagation d'une fissure existante. Le nœud $O$ correspond à la pointe de fissure. Les nœuds $A$ et $B$, situés sur le contour de la pièce, sont adjacents au nœud $O$. Le vecteur unitaire $\vec{u}$ représente la médiatrice des droites $O A$ et $O B$. La direction de la propagation de fissure est définie par le vecteur unitaire $\vec{v}$. La propagation de fissure n'est possible qu'à l'intérieur d'un domaine circulaire d'angle $2 \theta$ limité par les droites $\Delta_{1}$ et $\Delta_{2}$. A partir du critère de la contrainte circonférentielle maximale proposé par (Erdogan et al., 1963), Bouchard (2000) indique que l'angle maximal de bifurcation de la fissure est environ égal à $\pm 70^{\circ}$. C'est la valeur que nous retiendrons dans ces travaux pour l'angle $\theta$ (cf. figure 2a). Le paramètre $L$ est la longueur prédéfinie d'accroissement de fissure. Si la valeur $D_{\mathrm{C}}$ est atteinte en un point d'intégration de Gauss situé dans le secteur circulaire, alors la fissure se propage. Dans ce cas, pour chaque point de Gauss à 
l'intérieur du secteur circulaire, un vecteur unitaire donnant une direction «probable» de la pointe de fissure est défini. La direction «réelle» $\vec{v}$ de propagation de fissure est obtenue par une moyenne pondérée de chaque direction probable, d'une part, en fonction de la distance entre le point de Gauss et la pointe de fissure, et d'autre part, en fonction de la valeur du critère de rupture $D$ au point de Gauss considéré. Un nouveau nœud $P$, correspondant à la pointe de fissure actualisée, est alors ajouté au maillage comme le montre la figure $2 \mathrm{~b}$. Le nœud $O$ est ensuite divisé en deux nœuds afin d'ouvrir les lèvres de la fissure. Une fois les nouveaux nœuds créés, un remaillage complet est effectué ainsi qu'un incrément de rééquilibrage de la structure.

Dans l'approche de fissuration discrète proposée par (Brokken, 1999), la valeur de $D$ est évaluée en un nombre fini de points situés à plusieurs distances radiales par rapport à la pointe de fissure. Pour chaque distance radiale, une direction «probable» est établie pour laquelle la valeur de $D$ atteint un maximum. La direction « réelle» est obtenue en prenant la moyenne des directions «probables». Contrairement à la procédure de Brokken, la direction de propagation de fissure obtenue par notre approche de fissuration discrète est déterminée par une technique de double pondération : géométrique et intensité du champ $D$.

\section{Développements numériques}

\subsection{Logiciel de calcul par éléments finis}

Les simulations numériques ont été effectuées en utilisant le code de calcul par éléments finis BLANKFORM, qui a été développé pour simuler le procédé de découpage $2 \mathrm{D}$ et axisymétrique pour des comportements matériels thermo-élastoviscoplastiques en grandes déformations dans le cadre d'une formulation lagrangienne réactualisée. La résolution globale du problème d'équilibre est effectuée par un algorithme quasi statique implicite, dans lequel le problème non linéaire est remplacé par un système d'équations linéaires résolu par une méthode de type Newton-Raphson (Lemiale, 2004).

\subsection{Remaillage}

$\mathrm{Au}$ cours d'un processus de découpage, la tôle est soumise à de grandes déformations, ce qui peut conduire lors d'une simulation par éléments finis à une distorsion importante d'une partie des éléments. Afin de pallier ce problème, il est intéressant d'utiliser un algorithme de remaillage (Homsi et al., 1996; Wisselink, 2000), qui génère un nouveau maillage de la tôle chaque fois que cela est nécessaire. La qualité géométrique des éléments est alors conservée tout au long du calcul, ce qui permet de préserver la qualité des résultats numériques. 
L'algorithme de remaillage proposé utilise le mailleur BAMG, développé par Frédéric Hetch ${ }^{1}$, permettant de générer un maillage composé d'éléments triangulaires. Sachant que le temps de remaillage est négligeable par rapport à celui de la résolution, nous choisissons de réaliser un remaillage complet de la tôle. Le remaillage est activé, soit par un critère de périodicité à partir d'une fréquence définie à l'avance, soit par un critère géométrique basé sur une valeur seuil d'angle dans les éléments.

Une opération importante dans la mise en œuvre d'un algorithme de remaillage concerne le transport des champs calculés aux nœuds et aux points de Gauss (Lemiale, 2004). Il est important de remarquer que nous avons utilisé des éléments finis triangulaires à 6 nœuds et à 6 points de Gauss. Ce choix de sur-intégration est dû au transport des champs calculés des points de Gauss vers les nœuds. Afin d'éviter tout lissage des résultats, le nombre de points de Gauss a été choisi égal au nombre de nœuds (Lemiale, 2004).

Après la phase de transfert des champs, la structure n'est évidemment plus en équilibre du fait des approximations effectuées. En particulier, le champ de contraintes obtenu est différent de celui avant remaillage. Il est donc nécessaire d'effectuer un pas de calcul «à vide », c'est-à-dire sans modification de chargement, ceci afin de rééquilibrer la structure.

\section{Traction d'une plaque asymétrique}

\subsection{Description du test}

L'objectif de ce test de validation est de comparer les deux méthodes de propagation implémentées, à savoir la méthode d'élimination des éléments et la méthode de fissuration discrète. Ce problème a été présenté initialement par (Brokken, 1999; Brokken et al., 2000). L'auteur utilise ce calcul pour valider sa propre méthode de fissuration discrète. $\mathrm{Ne}$ disposant pas d'informations expérimentales précises sur ce problème, on se limitera à une analyse qualitative. Les caractéristiques propres aux deux méthodes seront mises en évidence.

On considère une plaque carrée de côté $1 \mathrm{~mm}$ en déformations planes (cf. figure 3). Le côté supérieur et le côté gauche de la plaque, dessinés en trait gras sur la figure 3 , sont soumis à un déplacement imposé dans la direction $y$. Les côtés inférieur et droit sont encastrés. Brokken indique que la géométrie particulière de ce problème, avec deux entailles de dimensions différentes, permet d'obtenir un trajet de fissure courbe qui résulte du développement d'une bande de cisaillement diagonale entre les deux entailles (Brokken, 1999).

1. Disponible sur le site http://www-rocq1.inria.fr/gamma/cdrom/www/bamg/fra.htm 


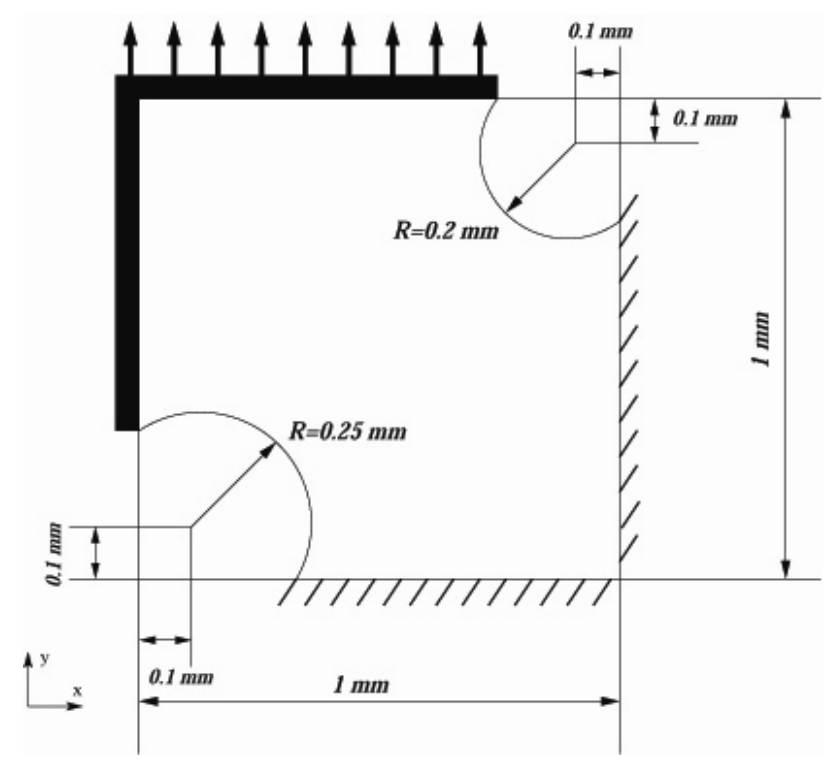

Figure 3. Géométrie initiale et conditions aux limites du problème d'une plaque asymétrique en traction (Brokken, 1999)

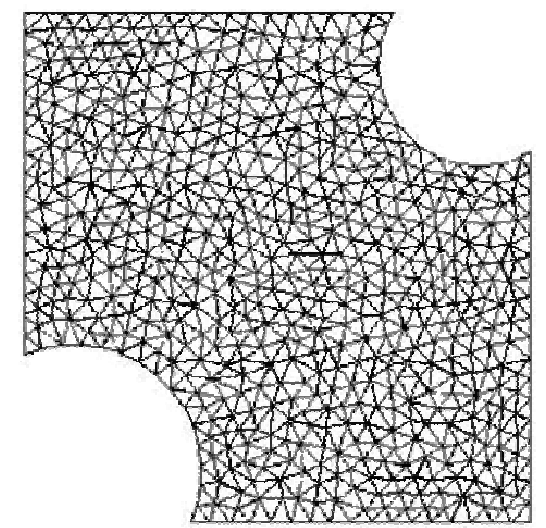

Figure 4. Maillage initial de la plaque

Le maillage initial de la plaque est représenté sur la figure 4. La taille moyenne de maille est choisie égale à $0,04 \mathrm{~mm}$. Le maillage est composé de 1172 éléments triangulaires à 6 nœuds et de 2443 nœuds. Le maillage choisi est volontairement 
assez grossier pour diminuer le temps de calcul. Par ailleurs, les caractéristiques des méthodes de rupture seront clairement mises en évidence sur ce type de maillage.

Le matériau utilisé est un acier inoxydable X30Cr13 dont les propriétés mécaniques sont consignées dans le tableau 2 (Goijaerts, 1999 ; Brokken, 1999). La courbe d'écrouissage est définie par l'équation suivante :

$$
\sigma_{0}=\sigma_{y}+h_{1}\left[1-\exp \left(-\frac{\bar{\varepsilon}^{\mathrm{p}}}{h_{2}}\right)\right]+h_{3} \sqrt{\bar{\varepsilon}^{\mathrm{p}}}
$$

\begin{tabular}{|l|c|}
\hline Paramètre & Valeur \\
\hline Module de Young, $E$ & $180000 \mathrm{MPa}$ \\
\hline Coefficient de Poisson, $v$ & 0,28 \\
\hline Limite initiale d'élasticité, $\sigma_{y}$ & $138 \mathrm{MPa}$ \\
\hline Constante du matériau, $h_{1}$ & $119 \mathrm{MPa}$ \\
\hline Constante du matériau, $h_{2}$ & 0,027 \\
\hline Constante du matériau, $h_{3}$ & $520 \mathrm{MPa}$ \\
\hline
\end{tabular}

Tableau 2. Caractéristiques mécaniques de l'acier inoxydable X30Cr13

Afin de parvenir à un critère de rupture adapté à l'acier X30Cr13, Goijaerts (1999) a évalué la capacité d'un certain nombre de critères à prédire l'amorçage de fissure pour un essai de découpage axisymétrique. Goijaerts a alors proposé une version adaptée du critère de (Oyane et al, 1980). Nous utiliserons finalement le même critère de rupture que (Brokken, 1999), qui prend la forme suivante :

$$
D=\int_{0}^{\bar{\varepsilon}^{\mathrm{p}}(t)}\left\langle 1+3 \frac{\sigma_{\mathrm{m}}}{\bar{\sigma}}\right\rangle \mathrm{d} \bar{\varepsilon}^{\mathrm{p}} \leq D_{\mathrm{C}} \quad \text { avec } \quad\langle a\rangle= \begin{cases}0 & \text { si } a \leq 0 \\ a & \text { si } a>0\end{cases}
$$

où $D_{\mathrm{C}}=2,4$ pour le matériau utilisé.

Pour cet essai, l'algorithme quasi statique implicite est utilisé. Le chargement est décomposé en incréments de déplacement de $2,510^{-3} \mathrm{~mm}$. Le remaillage n'est activé que lors de l'étape de propagation d'une fissure ou d'élimination d'un élément. La taille de maille moyenne imposée lors du remaillage est la même que pour le 
maillage initial, soit 0,04 mm, excepté pour les zones situées autour d'une fissure. La taille de maille choisie pour ces zones sera indiquée par la suite.

\subsection{Prédiction de l'amorçage d'une fissure}

La distribution au moment de l'amorçage d'une fissure de la valeur du critère d'endommagement défini précédemment est représentée sur la figure 5.

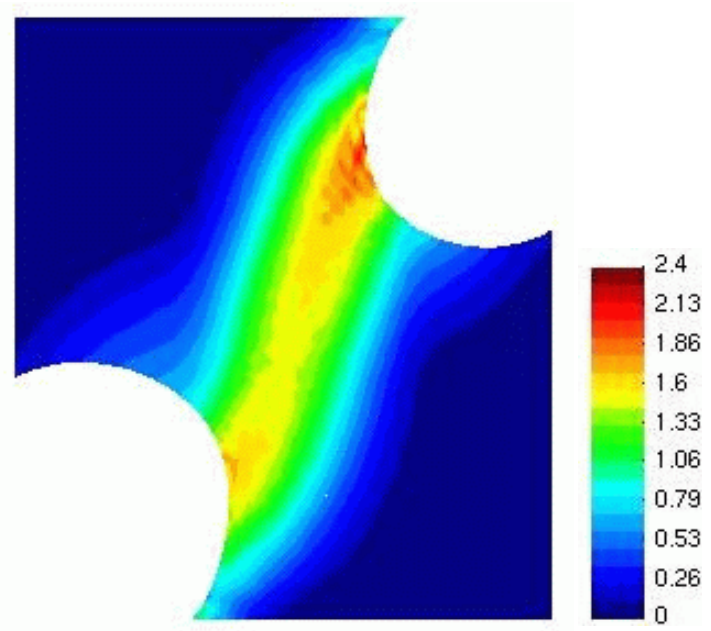

Figure 5. Distribution de la valeur du critère d'endommagement pour un déplacement égal à 0,12 $\mathrm{mm}$ (allongement de $12 \%$ )

Cette distribution est similaire à celle obtenue par Brokken. Le lieu d'amorçage de la première fissure se situe sur l'entaille de plus petit rayon, c'est-à-dire celle située dans le coin supérieur droit. L'amorçage est détecté pour un déplacement de $0,12 \mathrm{~mm}$, contre $0,11 \mathrm{~mm}$ obtenu par Brokken. Enfin, on observe que la trajectoire correspondant à la zone de plus fort endommagement correspond à une trajectoire courbe dirigée d'une entaille à l'autre. Là encore, les résultats concordent qualitativement avec ceux présentés par Brokken.

A partir de ce déplacement, on envisage l'étude de la rupture par la méthode d'élimination des éléments, puis par la méthode de fissuration discrète.

\subsection{Méthode d'élimination des éléments}

Le paramètre à définir lors de l'étape d'élimination d'un élément est la taille de maille pour la partie du contour définie par l'élément supprimé. Cette taille est fixée 
à $0,005 \mathrm{~mm}$ contre $0,04 \mathrm{~mm}$ initialement. Les résultats obtenus pour des déplacements respectifs de $0,13 \mathrm{~mm}, 0,138 \mathrm{~mm}$ et $0,142 \mathrm{~mm}$ sont présentés sur la figure 6. On observe que la fissure a tendance à se propager dans la direction de la seconde entaille. Les résultats exposés par Brokken ne concernent que le début de la propagation de la fissure. Le trajet de propagation présenté sur la figure 6 est similaire à celui obtenu par Brokken.

a)

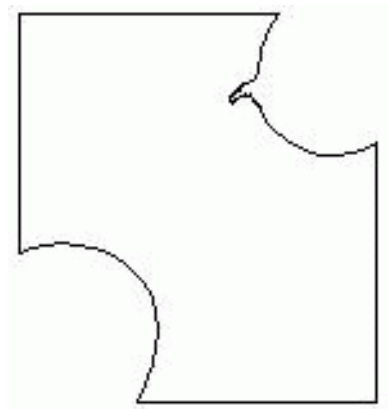

b)

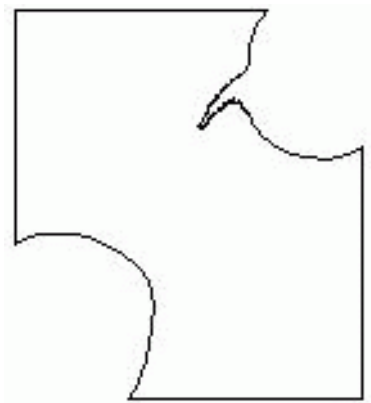

c)

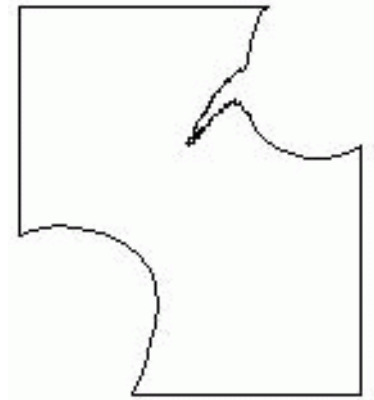

Figure 6. Propagation de fissure obtenue par élimination des éléments déplacement de : 0,13 $\mathrm{mm} \mathrm{a}$ ); 0,138 $\mathrm{mm} \mathrm{b}$ ); 0,142 $\mathrm{mm} \mathrm{c}$ )

On peut toutefois noter la dépendance au maillage éléments finis de la méthode d'élimination des éléments. Le maillage initial n'est pas suffisamment fin au niveau des entailles. Ceci entraîne, au début du calcul de propagation, une approximation assez grossière des dimensions de la fissure (cf. figure 6a). Lorsque la fissure se propage, les dimensions de la zone supprimée sont plus réduites (cf. figures $6 \mathrm{~b}$ et 6a). Ceci est dû au raffinement du maillage en pointe de fissure.

La taille du maillage est également critique en ce qui concerne la convergence de l'algorithme implicite. La suppression du premier élément de taille $0,04 \mathrm{~mm}$ rend difficile le retour à l'équilibre global de la structure. La convergence est obtenue après une trentaine d'itérations contre 6 ou 7 pour un incrément de chargement sans propagation de fissure. Lorsque les éléments supprimés ont pour taille de maille $0,005 \mathrm{~mm}$, le retour à l'équilibre est obtenu en une douzaine d'itérations.

Compte tenu du caractère approximatif de la solution pour ce maillage éléments finis, nous choisissons de ne pas mener le calcul à son terme par cette méthode. Notons cependant que le trajet de propagation est qualitativement cohérent avec celui obtenu par Brokken. 


\subsection{Méthode de fissuration discrète}

Pour la méthode de propagation de fissures discrètes, les deux paramètres à fixer sont la taille de l'accroissement d'une fissure $L$ et la taille de maille $t_{\mathrm{m}}$ en pointe de fissure. Pour limiter le temps de calcul, nous fixons $L$ à $0,02 \mathrm{~mm}$, et $t_{\mathrm{m}}$ égale à $L$. L'influence de ces deux paramètres sera ensuite discutée.

\subsubsection{Etude du trajet de fissuration}

La simulation du trajet de propagation des fissures est présentée sur la figure 7 . La rupture complète de la plaque survient pour un déplacement de $0,148 \mathrm{~mm}$. Les résultats fournis par Brokken ne concernent que la première partie du calcul (cf. figure 7a). Le trajet de propagation est similaire. L'angle d'inclinaison de la fissure par rapport à l'horizontale est cependant plus marqué pour (Brokken, 1999). En l'absence d'éléments de comparaison sur la suite du calcul, nous nous limitons à décrire les résultats obtenus et les caractéristiques de notre méthode de propagation de fissures. Pour un déplacement de $0,146 \mathrm{~mm}$, une seconde fissure est amorcée sur la seconde entaille (cf. figure 7b). Les deux fissures vont alors rapidement se propager en direction l'une de l'autre (cf. figure 7c). La rupture complète n'est pas simulée car l'algorithme proposé ne permet pas de gérer deux contours disjoints.

a)

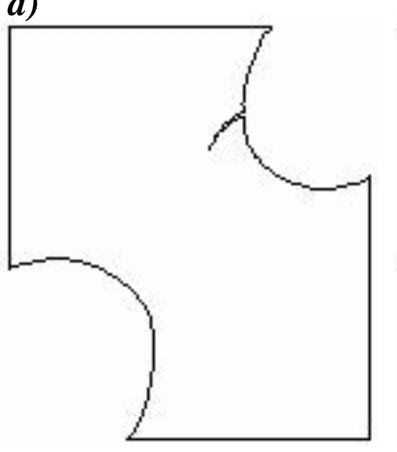

b)

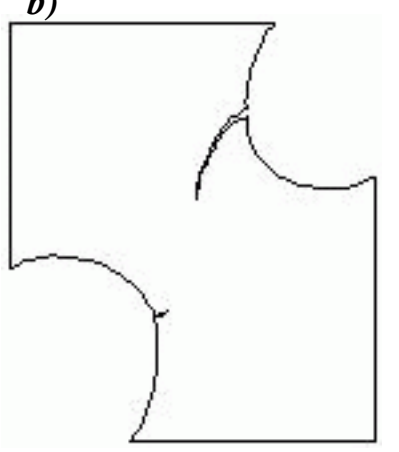

c)

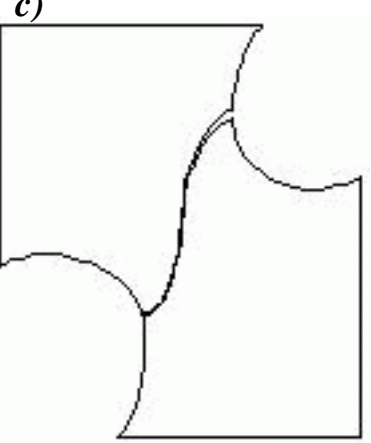

Figure 7. Propagation de fissure obtenue par la méthode de fissuration discrète déplacement de : 0,138 $\mathrm{mm} \mathrm{a)} \mathrm{;0,146} \mathrm{mm} \mathrm{b}) ; 0,148 \mathrm{~mm} \mathrm{c}$ )

\subsubsection{Prédiction de la variable d'endommagement}

La distribution de la variable d'endommagement calculée à partir du critère de type Oyane est présentée sur la figure 8. On constate que les deux fissures se sont effectivement propagées dans la direction d'endommagement maximal. Sur cette figure, certains éléments satisfont au critère de rupture (valeur d'endommagement égale à 2.86 pour une valeur critique à rupture de 2.4.) Ceci est dû au fait que ces 
éléments se situent sur les lèvres d'une fissure, et que l'amorçage d'une nouvelle fissure dans cette zone est interdit par la méthode.

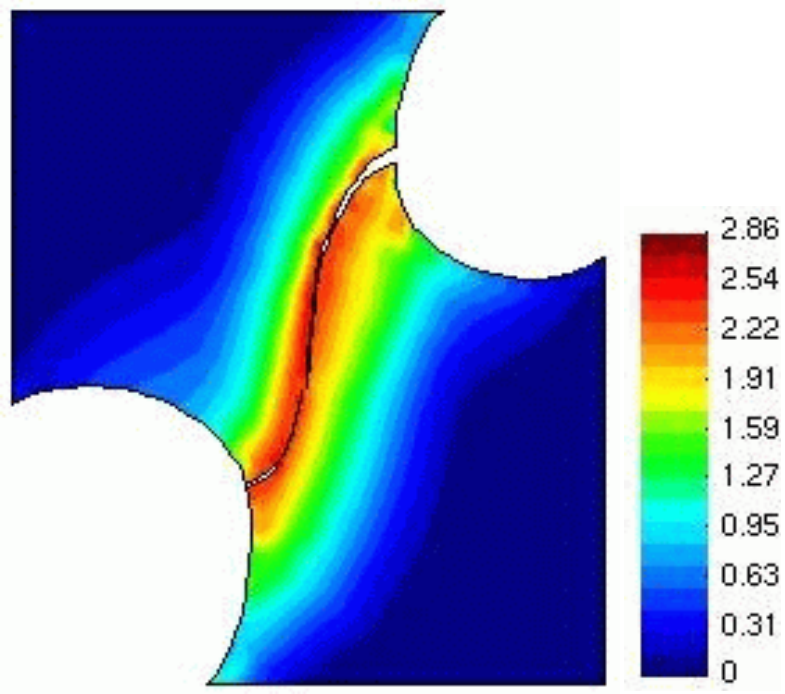

Figure 8. Distribution de la fonction critère d'endommagement $D$ pour un déplacement égal à $0,148 \mathrm{~mm}$ obtenu par fissuration discrète

\subsubsection{Influence des paramètres géométriques de fissuration}

Sur la figure 9, l'influence du paramètre d'accroissement de fissure $L$ sur le trajet de propagation est présentée en fixant la valeur du paramètre $t_{\mathrm{m}}$ à $0,02 \mathrm{~mm}$. On constate que les deux trajets obtenus pour $L=0,02$ et $L=0,06$ sont qualitativement comparables. Le trajet calculé avec $L=0,06 \mathrm{~mm}$ est cependant plus irrégulier que celui obtenu avec $L=0,02$. Ceci s'explique par le fait que l'augmentation de la longueur $L$ entraîne une discrétisation plus grossière de la fissure étudiée. Un autre calcul a été effectué avec une longueur $L=0,01 \mathrm{~mm}$. Les résultats sont très proches de ceux obtenus avec $L=0,02 \mathrm{~mm}$. Il est néanmoins difficile de fixer une règle générale pour la détermination de la longueur $L$ optimale. Celle-ci est à déterminer en fonction du problème étudié.

L'influence de la taille de maille $t_{\mathrm{m}}$ sur le trajet de propagation est maintenant illustrée sur la figure 10 en fixant la valeur du paramètre $L$ à 0,02 . On remarque que l'inclinaison de la fissure vers la seconde entaille est plus marquée pour $t_{\mathrm{m}}=$ $0,04 \mathrm{~mm}$ que pour $t_{\mathrm{m}}=0,02$. Ceci vient du fait que moins d'éléments interviennent dans le calcul de la direction de propagation. Par conséquent, l'influence des éléments situés dans la zone la plus endommagée est moins pondérée que pour $t_{\mathrm{m}}$ $=0,02 \mathrm{~mm}$. On peut cependant noter que même pour une taille d'élément grossière 
en pointe de fissure ( 8 fois plus importante que pour la méthode d'élimination des éléments), le trajet de propagation est qualitativement cohérent. La diminution de la taille $t_{\mathrm{m}}$ en pointe de fissure a également été étudiée. Pour $t_{\mathrm{m}}=0,5 \mathrm{~L}$, le trajet de propagation est très similaire à celui obtenu avec $t_{\mathrm{m}}=L$. La détermination précise de la taille de maille optimale en pointe de fissure nécessiterait une étude plus approfondie. Nous retiendrons pour les problèmes suivants le rapport $t_{\mathrm{m}} / L$ $=1$ comme rapport maximal à utiliser.

a)

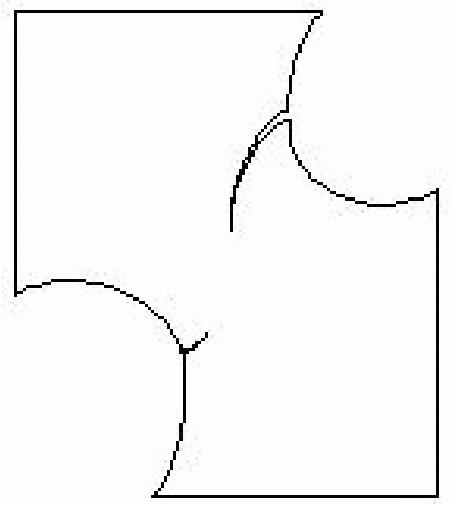

b)

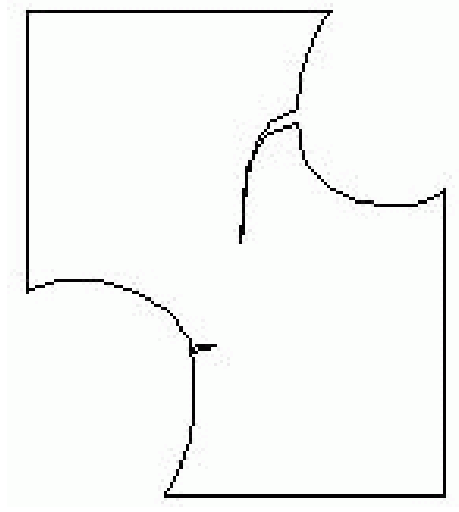

Figure 9. Comparaison du trajet de propagation pour un accroissement de fissure $L$ de : 0,02 $\mathrm{mm} \mathrm{a}$; $0,06 \mathrm{~mm}$ b) avec $t_{\mathrm{m}}=0,02 \mathrm{~mm}$

a)

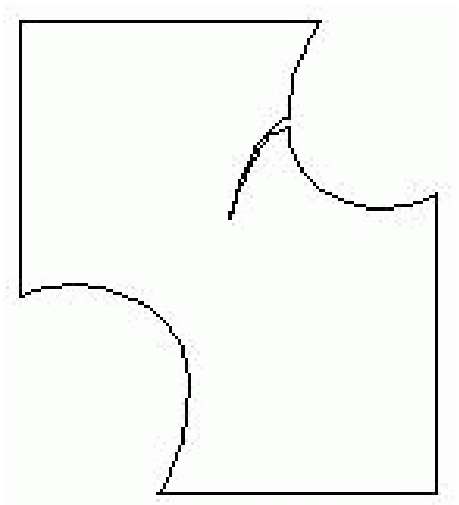

b)

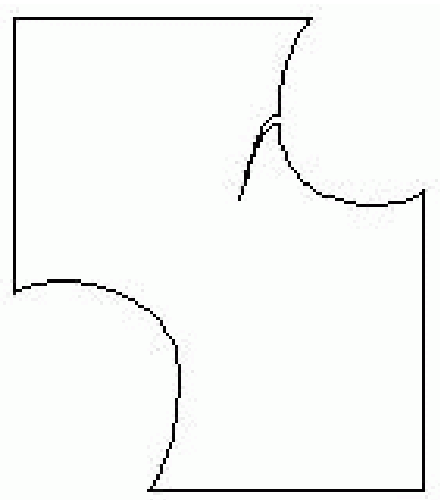

Figure 10. Comparaison du trajet de propagation pour une taille de maille $t_{\mathrm{m}}$ en pointe de fissure de : 0,02 $\mathrm{mm} \mathrm{a}$ ); 0,04 $\mathrm{mm} \mathrm{b}$ ) avec $L=0,02 \mathrm{~mm}$ 
Sur cet exemple, la méthode de fissuration discrète s'avère moins contraignante que la technique d'élimination des éléments pour la convergence de l'algorithme implicite. Le retour à l'équilibre est obtenu en une dizaine d'itérations. Le calcul est mené à son terme en $52 \mathrm{~min}$ pour les paramètres $L=0,02 \mathrm{~mm}$ et $t_{\mathrm{m}}=0,02 \mathrm{~mm}$ (sur un processeur pentium 4 cadencé à $2.4 \mathrm{GHz}$ ), en 34 min pour $L=0,06 \mathrm{~mm}$ et $t_{\mathrm{m}}$ $=0,02 \mathrm{~mm}$, et en $38 \min$ pour $L=0,02 \mathrm{~mm}$ et $t_{\mathrm{m}}=0,04 \mathrm{~mm}$.

\subsection{Premier bilan sur les méthodes de fissuration}

L'élimination des éléments rompus présente une importante dépendance vis-à-vis du maillage par éléments finis. De plus, la suppression d'un élément perturbe de manière significative l'équilibre de la structure. Par conséquent, il est indispensable de coupler ce type de méthode avec un maillage suffisamment fin. Cette technique est donc à utiliser avec précaution. Néanmoins, elle permet d'obtenir des résultats qualitativement cohérents.

Les résultats obtenus par la méthode de propagation de fissures discrètes sont similaires à ceux présentés par Brokken. La fissure amorcée sur l'entaille de plus petit rayon se propage en direction de la seconde entaille selon une trajectoire courbe. Seul le début de propagation est présenté par Brokken. En l'absence d'autres éléments de comparaison, on ne peut conclure quant au trajet complet de rupture simulé. Cependant, celui-ci semble réaliste, dans la mesure où les deux fissures amorcées se propagent en direction l'une de l'autre pour se rejoindre et aboutir à la rupture de la plaque. Enfin, on peut noter la robustesse numérique de la méthode de propagation de fissures. D'une part, la convergence du calcul est peu affectée par l'étape de propagation. La convergence est obtenue en une dizaine d'itérations contre 6 ou 7 sans calcul de rupture. D'autre part, l'influence du maillage en pointe de fissure est beaucoup moins prononcée que pour la méthode d'élimination des éléments. Cette technique semble donc bien adaptée à la simulation de la rupture ductile.

\section{Découpage axisymétrique}

\subsection{Description du test}

Les méthodes proposées d'amorçage et de propagation de fissure sont appliquées pour simuler un test de découpage axisymétrique et confrontées aux données expérimentales provenant de (Maillard, 1991). La géométrie et le maillage initial de la tôle sont présentés sur la figure 11a. Compte tenu de la présence d'un axe de symétrie, seul une moitié du problème est discrétisée ici. Le poinçon se situe sur le côté gauche supérieur; alors que la matrice et le serre flan sont sur le côté droit, respectivement inférieur et supérieur. La figure $11 \mathrm{~b}$ représente un zoom de la zone de localisation de la déformation située entre les arêtes du poinçon et de la matrice. 

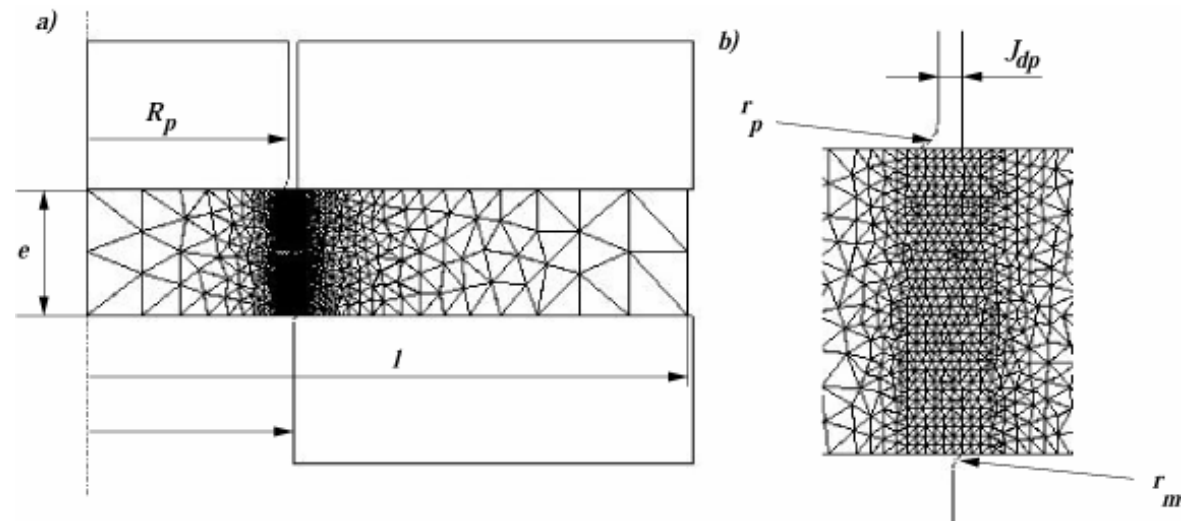

Figure 11. a) Géométrie des outils et maillage initial ; b) agrandissement de la zone de cisaillement

\begin{tabular}{|l|c|}
\hline \multicolumn{1}{|c|}{ Paramètre } & Valeur (en mm) \\
\hline Rayon poinçon, $R_{p}$ & 4 \\
\hline Rayon matrice, $R_{m}$ & 4,125 \\
\hline Rayon arête poinçon, $r_{p}$ & 0,225 \\
\hline Rayon arête matrice, $r_{m}$ & 0,15 \\
\hline Epaisseur tôle, $e$ & 2,5 \\
\hline Jeu poinçon-serre flan, $J_{d p}$ & 0,2 \\
\hline Rayon tôle, $l$ & 12 \\
\hline
\end{tabular}

Tableau 3. Caractéristiques géométriques du test de découpage axisymétrique

Les principales caractéristiques géométriques du modèle sont résumées dans le tableau 3. Pour les besoins de la simulation, les outils (la matrice, le poinçon et le serre flan) sont considérés comme des solides rigides indéformables et ils sont représentés uniquement par leurs contours (voir figure 11a). Dans les simulations proposées, le traitement du contact est effectué sur la base d'un glissement parfait entre la tôle et les outils. Le chargement est décomposé en incréments égaux de déplacement du poinçon de $2,510^{-3} \mathrm{~mm}$. Le maillage initial de la tôle est composé 
de 1342 éléments triangulaires à 6 nœuds et 6 points de Gauss. Dans la zone affinée du maillage, une taille de maille de $0,075 \mathrm{~mm}$ est conservée tout au long du calcul. Le remaillage de la tôle est effectué périodiquement, tous les $3 \%$ de déplacement du poinçon. Pour l'approche de fissuration discrète, l'accroissement $L$ de fissure a été choisi égal à $0,015 \mathrm{~mm}$ (Lemiale, 2004).

\subsection{Comportement du matériau}

Le matériau de la tôle découpée est un acier de qualité XES dont les propriétés mécaniques sont données dans le tableau 4. Sachant que les essais expérimentaux ont été réalisés avec une vitesse poinçon de $5 \mathrm{~mm} / \mathrm{min}$, les effets thermiques et visqueux ont été négligés dans les simulations numériques. Le comportement du matériau considéré est élastoplastique et la contrainte d'écoulement effective $\sigma_{0}$ suit la loi d'écrouissage suivante :

$$
\sigma_{0}=\sigma_{y}+k\left(\bar{\varepsilon}^{\mathrm{p}}\right)^{n}
$$

\begin{tabular}{|l|c|}
\hline \multicolumn{1}{|c|}{ Paramètre } & Valeur \\
\hline Module de Young, $E$ & $210000 \mathrm{MPa}$ \\
\hline Coefficient de Poisson, $v$ & 0,29 \\
\hline Limite initiale d'élasticité, $\sigma_{y}$ & $150 \mathrm{MPa}$ \\
\hline Constante du matériau, $k$ & $448 \mathrm{MPa}$ \\
\hline Exposant d'écrouissage, $n$ & 0,406 \\
\hline
\end{tabular}

Tableau 4. Propriétés mécaniques de l'acier XES

Le critère de rupture retenu est celui de Freudenthal (voir tableau 1). La valeur critique à rupture $D_{\mathrm{C}}$ du critère de Freudenthal a été identifiée numériquement de sorte que l'amorçage de fissure simulé par éléments finis corresponde au niveau de pénétration obtenu expérimentalement par Maillard (Maillard, 1991), soit $76 \%$ de l'épaisseur. Une valeur de 1,36 a ainsi été obtenue pour $D_{\mathrm{C}}$. 


\subsection{Résultats numériques}

La figure 12a illustre le profil à rupture du contour déterminé par la méthode d'élimination des éléments pour une pénétration du poinçon de $89 \%$ de l'épaisseur. Le profil obtenu par l'approche de fissuration discrète est présenté sur la figure $12 \mathrm{~b}$ pour une pénétration du poinçon de $82 \%$. En comparant les figures $12 \mathrm{a}$ et $12 \mathrm{~b}$, nous constatons, comme précédemment, que le profil de découpe obtenue par la méthode d'élimination des éléments présente une plus forte sensibilité au maillage que celui simulé par l'approche de fissuration discrète. Cela peut s'expliquer par le fait que la méthode d'élimination consiste à enlever de la matière. D'où, cette technique dépend fortement de la taille et de la forme des éléments finis dans la zone de cisaillement. Le manque d'informations précises sur l'évolution du profil de rupture expérimental (Maillard, 1991) ne nous permet pas de confronter à la réalité les simulations du profil obtenues par ces deux méthodes.

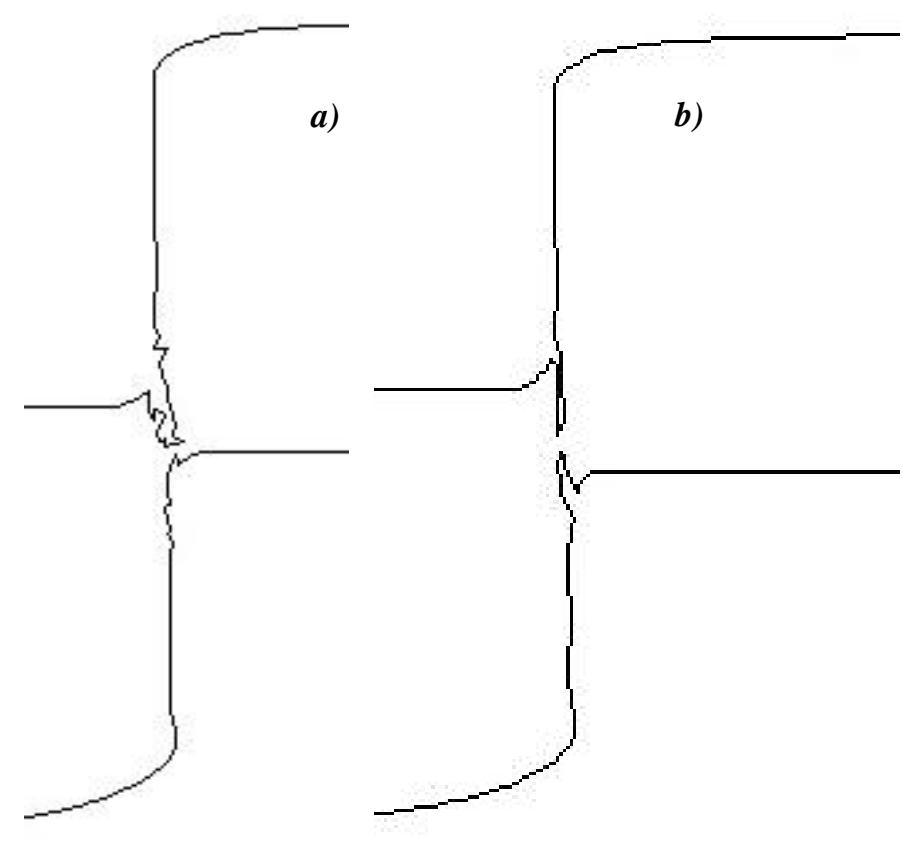

Figure 12. Profils de découpe: a) méthode d'élimination des éléments; b) approche de fissuration discrète 

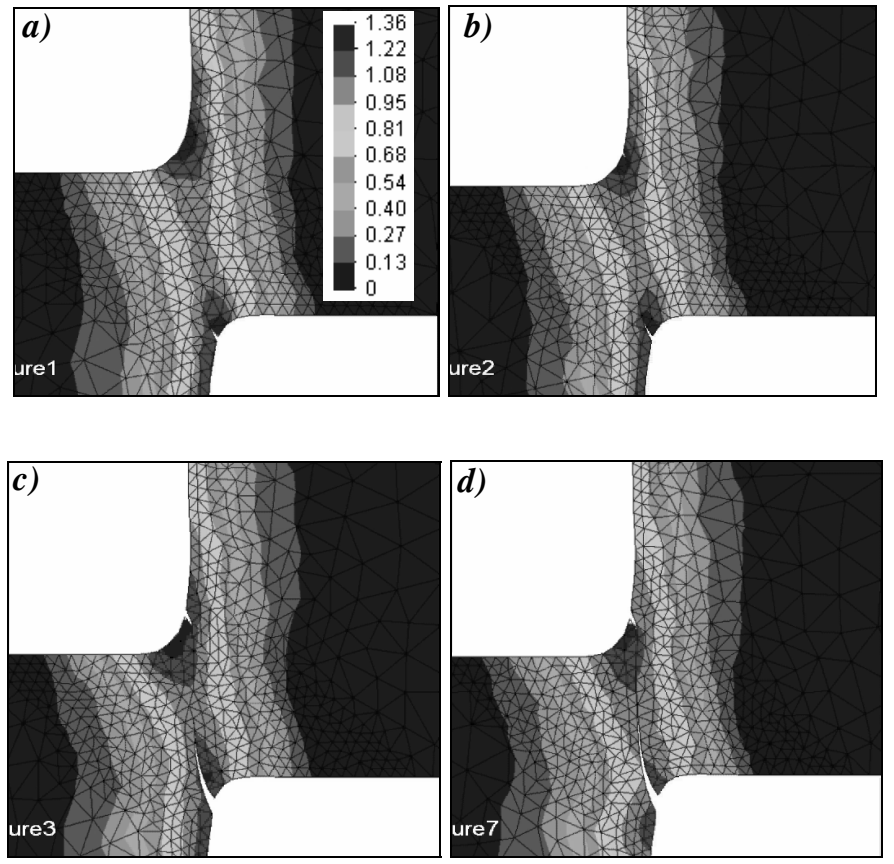

Figure 13. Evolution de la variable d'endommagement $D$ avec l'approche de fissuration discrète

L'évolution du champ d'endommagement pendant la propagation de fissure est présentée sur la figure 13 en utilisant l'approche de fissuration discrète. On peut voir qu'une première fissure s'amorce au niveau de l'arête de la matrice pour une pénétration du poinçon de $76 \%$ (figure 13a), puis une seconde fissure apparaît près de l'arête du poinçon (figure 13b). Finalement, les deux fissures se propagent rapidement et de manière réaliste l'une vers l'autre (figures 13c et 13d). Il semble donc que les phases d'initiation et de propagation des fissures sont correctement simulées par l'approche de fissuration discrète.

La figure 14 présente les courbes effort-déplacement du poinçon expérimentale et numériques obtenues par les deux méthodes de propagation de fissures. La courbe effort-déplacement expérimentale est correctement prédite par les deux modèles. On observe que les deux courbes obtenues par simulation numérique coïncident jusqu'à un déplacement de $1,8 \mathrm{~mm}$. Puis, la chute de la résistance mécanique est simulée plus précisément par l'approche de fissuration discrète que par la méthode d'élimination des éléments. Les résultats peuvent être améliorés par une meilleure identification de la valeur critique d'endommagement $D_{\mathrm{C}}$ (Lemiale, 2004). La surévaluation de l'effort du poinçon peut être réduite en utilisant une approche 
couplée des équations constitutives qui prenne en compte la perte des propriétés mécaniques due à l'endommagement (Lemiale, 2004).

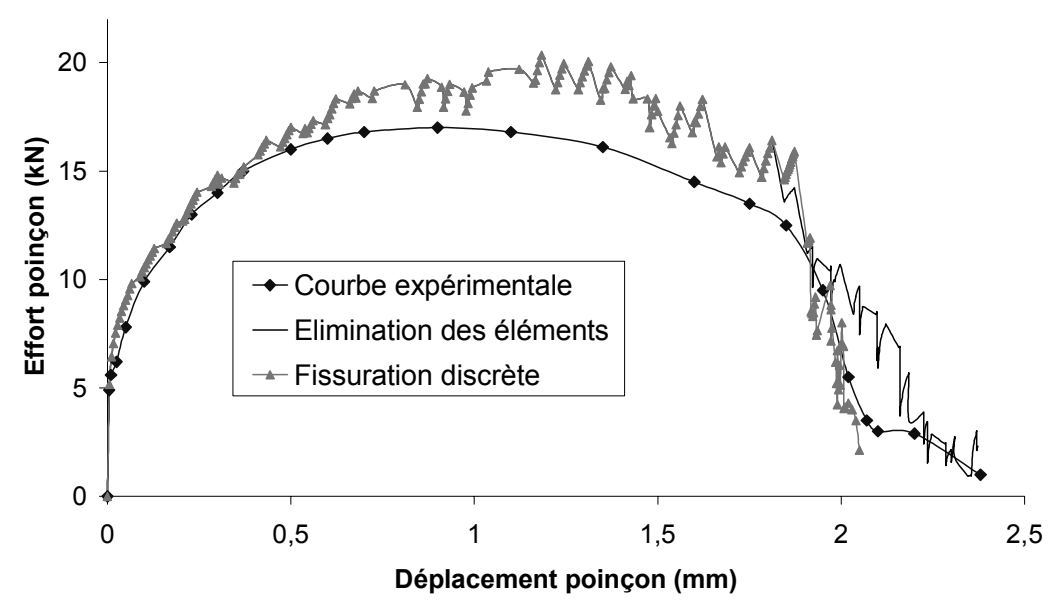

Figure 14. Comparaison des courbes d'effort-déplacement du poinçon expérimentales et calculées par les méthodes de propagation de fissures

\section{Conclusion}

Un logiciel de calculs par éléments finis, appelé BLANKFORM, a été développé pour simuler le procédé de découpage de tôle. Dans l'approche proposée, l'amorçage de fissure est prédit par un critère de rupture. Deux méthodes de propagation de fissure ont été proposées et implémentées : la méthode d'élimination des éléments et l'approche de fissuration discrète. L'analyse des résultats obtenus par ces deux méthodes sur le problème d'une plaque asymétrique en traction a montré que la technique d'élimination des éléments présente une plus forte dépendance au maillage que l'approche de fissuration discrète.

Concernant le découpage axisymétrique, les résultats obtenus ont montré un assez bon accord avec les données expérimentales en termes de courbe effortdéplacement du poinçon quel que soit la méthode de fissuration utilisée. Cependant, l'approche de fissuration discrète fournit une meilleure prédiction du profil de la pièce découpée que la méthode d'élimination des éléments. L'outil de simulation numérique développé permet d'obtenir en outre des prédictions intéressantes en termes de déformation plastique équivalente, de contraintes et d'endommagement permettant de caractériser les propriétés mécaniques de la pièce découpée. 


\section{Remerciements}

Les auteurs remercient la société AUGE Découpage pour sa collaboration et son soutien financier.

\section{Bibliographie}

Bouchard P.-O., Contribution à la modélisation numérique en mécanique de la rupture et structures multimatériaux, Thèse de doctorat, Ecole des Mines de Paris, 2000.

Bouchard P.-O., Bay F., "Damage and crack propagation theories applied to sheet metal cutting", Proc. of the $14^{\text {th }}$ European Conference on Fracture ECF14, Cracow, Poland, September 8-13, ESIS/ASTM, EMAS, 2002.

Brokken D., Numerical modelling of ductile fracture in blanking, $\mathrm{PhD}$ Thesis, Eindhoven University of Technology, The Netherlands, 1999.

Brokken D., Brekelmans W.A.M., Baaijens F.P.T., "Discrete ductile fracture modelling for the metal blanking process", Comp. Mech., vol. 26, 2000, p. 104-114.

Cockroft M.G., Latham D.J., "Ductility and the workability of metals", J. Inst. Met., vol. 96, 1968, p. 33-39.

Erdogan F., Sih G.C., "On the crack extension in plates under plane loading and transverse shear", J. Basic Eng., vol. 85, 1963, p. 519-527.

Fang G., Zeng P., Lou L., "Finite element simulation of the effect of clearance on the forming quality in the blanking process", J. Mater.Proc. Techn., vol. 122, 2002, p. 249-254.

Freudenthal A.M., The Inelastic Behaviour of Engineering Materials and Structures, John Wiley \& Sons, New York, 1950.

Goijaerts A.M., Prediction of ductile fracture in metal blanking, PhD Thesis, Eindhoven University of Technology, The Netherlands, 1999.

Hambli R., Etude expérimentale, numérique et théorique du découpage de tôles en vue de l'optimisation du procédé, Thèse de doctorat, ENSAM d'Angers, 1996.

Hatanaka N., Yamaguchi K., Takakura N., "Finite element simulation of the shearing mechanism in the blanking of sheet metal", J. Mater.Proc. Techn., vol. 139, 2003, p. 64-70.

Homsi M., Morançay L., Roëlandt J.-M., «Techniques de remaillage appliquées au découpage des métaux », Revue Europ. Eléments Finis, vol. 5, 1996, p. 297-321.

Ko D.C., Kim B.M., Choi J.C., "Finite element simulation of the shear process using the element-kill method", J. Mater.Proc. Techn., vol. 72, 1997, p. 129-140.

Komori K., "Simulation of shearing by node separation method", Comp. \& Struct., vol. 79, 2001, p. 197-207.

Komori K., "Ductile fracture criteria for simulating shear by node separation method", Theo. Appl. Frac. Mech., vol. 43, 2005, p. 101-114. 
Lemiale V., Contribution à la modélisation et à la simulation numérique du découpage des métaux, Thèse de doctorat, Université de Franche-Comté, Besançon, 2004.

Maillard A., Etude expérimentale et théorique du découpage, Thèse de doctorat, Université de Technologie de Compiègne, 1991.

McClintock F.A., "A criterion for ductile fracture by the growth of holes", J. Appl. Mech., vol. 35,1968 , p. 363-371.

Oyane M., Sato T., Okimoto K., Shima S., "Criteria for ductile fracture and their applications", J. Mech. Work. Tech., vol. 4, 1980, p. 65-81.

Rice J.R., Tracey D.M., "On the ductile enlargement of voids in triaxial stress fields", J. Mech. Phys. Solids, vol. 17, 1969, p. 201-217.

Taupin E., Breitling J., Wu W.T., Altan T., "Material fracture and burr formation in blanking results of FEM simulations and comparison with experiments", J. Mater. Proc. Techn., vol. 59, 1996, p. 68-78.

Wisselink H., Analysis of guillotining and slitting, finite element simulations, $\mathrm{PhD}$ Thesis, University of Twente, The Netherlands, 2000. 
